\title{
Lo Público y to Privado; las Aseguradoras y la Atención Médica en Mexico
}

\author{
Public and Private: Insurance Companies and Medical Care in Mexico
}

\author{
Silvia Tamez ${ }^{1}$ \\ Claudia Bodek ${ }^{2}$ \\ Catalina Eibenschutz ${ }^{1}$
}

\section{TAMEZ, S; BODEK, C. \& EIBENSCHUTZ, C. Public and Private: Insurance Companies and Medical Care in Mexico. Cad. Saúde Públ., Rio de Janeiro, 11 (4): 579-587, Oct/Dec, 1995.}

During the late 70's and early 80's in Mexico, as in the rest of Latin-America, sanitary policies were directed to support the growth of the private sector of health care at the expense of the public sector. This work analyzes the evolution of the health insurance market as a part of the privatization process of health care. The analysis based on economic data, provides the political profile behind the privatization process as well as the changes in the relations between the State and the health sector. The central hypothesis is that the State promotes and supports the growth of the private market of medical care via a series of legal, fiscal and market procedures. It also discusses the State roll in the legal changes related to the national insurance activity. A comparative analysis is made about the evolution of the insurance industry in Argentina, Brazil, Chile and Mexico during the period 1986-1992, with a particular enfasis in the last country. One of the principal results is that the Premium/GNP and Premium/per capita, display a general growth in the 4 countries. This growth is faster for Mexico for each one) because the privatization process ocurred only during the most recent years. For the 1984-1991 period in Mexico the direct premium as percentage of the GNP raised from $0.86 \%$ to $1.32 \%$. If one focussed only in the insurance for health and accidents branches the rice goes form $8.84 \%$ in 1984 to $19.08 \%$ in 1991. This indicates that the insurance industry is one of the main targets of the privatization process of the health care system in Mexico. This is also shown by the State support to fast expansion of the big medical industrial complex of the country. Considering this situation in the continuity of the neoliberal model of Mexico, this will profound the inequity and inequality.

Key words: Public; Private; Privatization; Medical Care; Medical Insurance

\section{INTRODUCCION}

En la década de los 80's, la mayoría de los países de América Latina experimentaron importantes cambios económicos, políticos y sociales los cuales han implicado

\footnotetext{
'Departamento de Atención a la Salud, Universidad Autónoma Metropolitana Xochimilco. Calzada del Hueso $N^{o}$. 1100. Col. Villa Quietud, 04960 D.F., Mexico.

${ }^{2}$ Academia de Historia de las Ideas, Universidad Pedagógica Nacional. Carretera al Ajusco No. 24. Col. Héroes de Padierna, 14200 D.F., Mexico.
}

transformaciones en el campo de los servicios de salud, caracterizadas por dos rasgos fundamentales: 1) recorte del gasto público y política de ajuste; y 2) aumento de la participación del sector privado en áreas que habían sido casi exclusivas del sector público.

Varios autores (Eibenschutz, 1991; Laurell, 1992) han planteado que el crecimiento de los seguros medicos privados en el país es hasta ahora el eje principal del proceso de crecimiento del sector privado de la atención médica. Esto que se relaciona con el aumento significativo en las camas privadas (cuyo registro es todavía muy impreciso), ya que, de 3,000, en 1985. pasó a 17,000, en 1991 (Soberón 
et al., 1989; WHO/SHS/NAP, 1991). Este crecimiento se ha visto acompañado de modiflcaciones legales promovidas por el Estado que abren espacio al sector privado.

El objetivo del trabajo es describir y analizar, como parte del proceso privatizador, la evolución de los seguros médicos privados en México durante el periodo 1986-1992, a partir de los cambios iniciados a principios de la década pasada. Para tal efecto, se consideró necesario analizar la situación mexicana dentro de un marco más amplio, que permitiera comparar la evolución de los seguros médicos en nuestro país con países relativamente similares, como Brasil, Chile y Argentina.

\section{PAPEL DEL ESTADO EN LAS TRANSFORMACIONES DEL SECTOR SALUD EN MEXICO}

Debido al giro "modernizador" adoptado por nuestra economía, se ha producido una desregularización generalizada de la normatividad que rige las diferentes relaciones económicas, situación que se aplica al mundo de los seguros y las fianzas. En este caso, el gobierno ha impulsado reformas y decretos que tienden a propiciar la apertura del sector en aras de lograr una mayor eficiencia y competitividad de las compañías de seguros y afianzadoras.

De acuerdo al discurso de las empresas aseguradoras (Ruíz-Ramirez, 1992; AMIS, 1991), dichas reformas están dirigidas fundamentalmente a eliminar barreras y fomentar la competencia a través de concesiones de carácter discrecional y a promover eficiencia, autorizando al sector extranjero su participación en el capital social de las aseguradoras, así como, crear grupos financieros a través de una ley especial para ello. Para lograr to anterior, se realizaron reformas a la nueva Ley General de Instituciones y Sociedades Mutualistas de Seguros, a partir de las se deja a las empresas aseguradoras en libertad de funcionamiento y prácticamente sin supervisión oficial (Ruíz-Ramirez, 1992). Las reformas también han sido orientadas a flexibilizar las funciones de las aseguradoras para incentivar el crecimiento de su mercado frente a las necesidades que impone el Tratado de Libre Comercio (TLC). Por otro lado, el gobierno mismo otorga diferentes tipos de seguro médico privado a sus trabajadores, garantizando un mercado dinámico para las empresas de seguros.

De esta manera el Estado cambia su carácter y de ser un Estado que fundamentalmente produce y otorga servicios de salud, incorpora como una de sus prioridades el generar condiciones para el desarrollo del libre mercado de la atención médica.

\section{LA INDUSTRIA ASEGURADORA EN CHILE, BRASIL, ARGENTINA Y MEXICO}

Dado que en la última década la privatización de los servicios de salud es un proceso compartido por la mayoría de los paises de América Latina, se seleccionaron algunos modelos paradigmáticos, tanto por su historia sanitaria como por sus formas de articulación y crecimiento de la industria de los seguros médicos privados.

\section{Análisis Comparativo}

En los cuadros 1 y 2 se presenta el crecimiento del PIB en los cuatro paises durante el período estudiado y la evolución de las primas/PIB, en miles de dólares, respectivamente.

Hasta 1990, los cuatro países muestran un crecimiento sostenido en el indicador primas/ PIB. Sin embargo, entre 1990 y 1992, en Argentina, se observa una caída muy importante $(-50.93 \%)$, lo que no forzosamente significa un decremento real ya que el PIB creció durante ese periodo de manera espectacular, pues en 1990 fue de $-0.20 \mathrm{y}$, para 1992, ascendió a 8.70, (Cuadro 1).

Brasil, por su parte, hasta 1990 es el país que ocupa el segundo lugar en crecimiento de primas/PIB, registrando entre 1988 y 1990 un aumento de $75.82 \%$, pero, entre 1990 y 1991(no se cuenta con el dato de 1992), se observa un 
CUADRO 1. Crecimiento del PIB, 1986-1992

\begin{tabular}{lrrrrrrr}
\hline \hline País & 1986 & 1987 & 1988 & 1989 & 1990 & 1991 & 1992 \\
\hline Argentina & 6 & 2,1 & $-2,8$ & $-4,5$ & $-0,2$ & 4,5 & 8,7 \\
Brasil & 7,5 & 3,7 & -3 & 3,6 & $-4,6$ & 0,9 & $-0,93$ \\
Chile & 5,5 & 4,9 & 7,6 & 9,3 & 2,1 & 6 & 10,4 \\
México & $-3,8$ & 1,7 & 1,4 & 3,1 & 4,6 & 4 & 2,2 \\
\hline \hline
\end{tabular}

CUADRO 2. Evolución de Primas/PIB en América Latina, Miles de Dólares, 1986-1992

\begin{tabular}{|c|c|c|c|c|c|c|c|c|c|}
\hline \multirow[b]{3}{*}{ País } & \multicolumn{2}{|c|}{1986} & \multicolumn{2}{|c|}{1988} & \multicolumn{2}{|c|}{1990} & \multicolumn{2}{|c|}{1992} & \multirow[b]{2}{*}{$\%$} \\
\hline & $\%$ & $\%$ & $\%$ & $\%$ & $\%$ & $\%$ & $\%$ & $\%$ & \\
\hline & Prim./PIB & Variación & Prim./PIB & Variación & Prim./PIB & Variación & Prim./PIB & Variación & Variación \\
\hline Argentina & 2,47 & 0 & 2,69 & 8,9 & 3,1 & 13,24 & 1,52 & $-50,93$ & $-3800 \%$ \\
\hline Brasil & 0,72 & & 0,91 & 26,38 & 1,6 & 75,82 & $0.41^{*}$ & $-74,37$ & $-43,05$ \\
\hline Chile & 1,93 & 0 & 2,17 & 12,93 & 2,78 & 32,25 & 4,04 & 45,32 & 109 \\
\hline México & 0,7 & 0 & 1,14 & 62,85 & 1,2 & 87 & 1,52 & 33,33 & 117 \\
\hline
\end{tabular}

brusco decremento de $-74.37 \%$, lo cual podrá tener la misma explicación que en el caso de Argentina, pues, en 1990, el crecimiento porcentual del PIB en relación a 1989 paso de -4.60 a 0.90 .

Chile, el país considerado prototípico de expansión acelerada de la atención médica privada, muestra un crecimiento sostenido en las primas/PIB, alcanzando su máximo en el bienio 1990-92, con 45.32\%. Analizando esta tendencia en contraste con el crecimiento porcentual del PIB en este país, encontramos que el crecimiento del indicador es válido ya que, a excepción de 1991, año en el que el PIB decrece, en general su crecimiento es constante (Cuadro 1 ).

Hasta 1990, México es el país en que la relación primas/PIB aumenta más, con un $62.85 \%$ entre 1986 y 1988 y un $87.00 \%$ entre 1988-90. Para el bienio de 1990-92, el crecimiento continua, aunque en menor proporción (33.33\%). El crecimiento de este indicador para nuestro país puede ser considerado como real, ya que el PIB creció sostenidamente a partir de 1987 y hasta 1990, observandose un descenso pronunciado en 1992 ya que, de 1990 a 1992, decreció un $51.4 \%$.
Analizando la totalidad del periodo, comparando 1986 y 1992, encontramos que Argentina y Brasil descienden $38.00 \%$ y $-43.05 \%$, respectivamente, to cual como ya se mencionó, es probable que se relacione con la evolución del PIB durante el periodo. Por su pane, Chile y México muestran un importante crecimiento de $109 \%$ y $117 \%$, respectivamente, en condiciones de crecimiento más o menos sostenido del PIB.

En términos generales, podemos decir que, en los cuatro países analizados, existe un incremento de los seguros privados durante el periodo de 1986-1992, a excepción del seguro de no vida en Argentina, el cual registra un decrecimiento de $37.93 \%$ (Cuadro 3 y 4 ).

Llaman la atención los aumentos tan pronunciados en los cuatro países en el seguro de vida, rubro dentro del cual se encuentran los seguros médicos, siendo México el país que presenta el porcentaje de mayor crecimiemo, el cual llega a ser seis veces mayor que en Chile y cerca de nueve veces más que el de Brasil. Este panorama es similar para el seguro de no vida. Esta situación puede ser explicada, en gran medida, porque, hasta antes de la segunda mitad de la década pasada, prácticamente 
no existía la industria de los seguros en nuestro país.

Relacionando este indicador con el crecimiento de la población en los cuatro países, habría que destacar que, en Brasil, la población aumentó mucho durante el periodo, incrementandose en 17,510,000 personas. En México, Argentina y Chile la población aumenta 4,680,000, 2,260,000 y cerca de 1,271,000, respectivamente. Al margen de considerar los procesos inflacionarios de los países analizados, estas cifras son sugerentes de que la industria de los seguros privados se encuentra en proceso de expansión, siendo más pronunciado dicho proceso en México.
A partir del análisis anterior, podemos hacer las siguientes reflexiones:

- Al comparar la evolución y el crecimiento de la industria aseguradora entre México y Chile (primas/PIB, primas per cápita), encontramos que el crecimiento entre 1986 y 1992 es más acelerado en México, to que sin duda se debe a que, en nuestro país, el proceso de privatización es muy incipiente comparado con Chile, país en el que la privatización está muy avanzada. Probablemente esto se deba también a la diferencia en los modelos de privatización y financiamiento existentes

CUADRO 3. Evolución de las Relacións Primas Per Cápita del Ramo de Vida y no Vida en América Latina, 1986-1992 (US\$)

\begin{tabular}{lcccccccc}
\hline \hline & \multicolumn{2}{c}{1986} & \multicolumn{2}{c}{1990} & \multicolumn{2}{c}{1992} & \multicolumn{2}{c}{$\%$} \\
País & Primas/PC & \% Variación & Primas/PC & \% Variación & Primas/PC & \% Variación & Variación \\
\hline Argentina & 58 & 0 & 80 & 37,93 & 36 & -55 & $-37,93$ \\
Brasil & 16 & 0 & 28 & 75 & 86 & 207,14 & 437,5 \\
Chile & 13 & 0 & 23 & 76,92 & 85 & 269,56 & 553,84 \\
México & 8 & 0 & 23 & 187,5 & 184 & 700 & 2200 \\
\hline \hline
\end{tabular}

CUADRO 4. Evolución de las Relacións Primas/PIB, Seguro de Vida en América Latina, 1986-1992 (US\$)

\begin{tabular}{lcccccccc}
\hline \hline & \multicolumn{2}{c}{1986} & \multicolumn{2}{c}{1990} & \multicolumn{2}{c}{1992} & \multicolumn{2}{c}{$\%$} \\
País & Primas/PIB & \% Variación & Primas/PIB & \% Variación & Primas/PIB & $\%$ Variación & Variación \\
\hline Argentina & 6 & 0 & 8 & 33,33 & 88 & 1000 & 1366,66 \\
Brasil & 3 & 0 & 4 & 33,3 & 30 & 650 & 900 \\
Chile & 13 & 0 & 34 & 161,53 & 180 & 430,29 & 1284,61 \\
México & 3,3 & 0 & 12 & 263,63 & 270 & 2150 & 8081,8 \\
\hline \hline
\end{tabular}

entre los dos países. Chile implantó el modelo de las Instituto de Salud Provisional en el año de 1983, to que significó el traspaso de las cuotas de los trabajadores al sector privado, a diferencia de México, dónde el crecimiento se da a partir de 1986, y hasta el momento, los seguros privados tienen una función complementaria respecto al público. En cambio, Brasil está en un momento diferente, debido a las características de su reforma sanitaria, que intenta priorizar la responsabilidad,del Estado y la universalización frente a la presión del modelo neoliberal. Además, dada la magnitud del proceso inflacionario y considerando el hechò de que las camas son privadas desde la década de los sesenta, parece no haber en ese país un crecimiento significativo en los seguros médicos durante los últimos años. 


\section{EVOLUCION DE LA INDUSTRIA ASEGURADORA EN MEXICO}

El sector asegurador de México está concentrado en empresas privadas; del total de 42 aseguradoras que integraban el sector hasta 1994, 37 eran empresas privadas, mientras que solo 3 eran del Estado y 2 de tipo mutualistas (AMIS, 1992).

Aseguradora Mexicana (ASEMEX), considerada como de las más importantes a nivel nacional, fue privatizada recientemente, lo que implica que el Estado mantendrá solamente dos instituciones de seguros: Agroasemex e Hidalgo.

Por otra parte, se observa el crecimiento de capitales en las empresas de seguros privados en los siguientes datos: hasta 1990, había cinco instituciones de seguro directo y una reaseguradora que tenía participación de capital extranjera (entre el 20 y el $44 \%$ ); hoy en día, hay inversión extranjera en nueve instituciones de seguro directo (AMIS, 1992). Existen autores que afirman que el sector asegurador es, junco con arrendadoras y factoraje, el más abierto a la inversión extranjera del sistema financiero mexicano (Gil-Anton \& Sólis-Soberon, 1991).

A pesar del fortalecimiento que las modificaciones legales referidas con anterioridad han causado al sector asegurador, éste las considera insuficientes para lograr su meta de crecimiento de llegar a representar el $2 \%$ del PIB, según acuerdo discrecional con el Presidente, por lo cual reclama más modificaciones legales. $\mathrm{Al}$ analizar el discurso del sector asegurador, queda claro que la universalidad de la cobertura médica es considerada de manera secundaria frente a la lógica del rendimiento económico que el sistema impone.
Siendo que los seguros representan un sector significativo del mercado financiero, los empresarios de las aseguradoras se plantean como necesario el tener un crecimiento mayor que el del PIB. Para ello su actividad se ha venido centrando básicamente en la ampliación de su mercado. De esta manera tenemos que, en los últimos años, el índice de crecimiento de primas directas reales ha sido significativo, ya que, de 1984 a 1991, tuvo un crecimiento del 57\%, mientras que el PIB en términos reales ha crecido, en ese mismo lapso de tiempo, en un 5\%.

Si consideramos a las primas directas de los seguros como porcentaje del PIB, para el período 1984-1991 tenemos que han pasado de $0.86 \%$ a representar el $1.32 \%$ (AMIS, 1991). Nos interesa rescatar la alta proporcionalidad de crecimiento y subrayar que, para ese mismo período de tiempo, los seguros

correspondientes a accidentes y enfermedades representaron del $0.02 \%$, en 1984 , el $0.11 \%$ del PIB en 1991 (Cuadro 5).

Como puede apreciarse en el siguiente cuadro, desde el punto de vista de la importancia de este tipo de seguro respecto al de vida, encontramos que el seguro de accidentes y enfermedades representaba, en 1984, el 8.84\% y, para 1993, dicha proporción ascendió a $19.08 \%$, to que nos está hablando de una tendencia consistente de aumento de los seguros médicos en nuestro país.

Ante la firma del Tratado de Libre Comercio con los Estados Unidos y Canadá, la industria aseguradora mexicana se ha planteado reforzar y ajustar un gran número de rubros de sus operaciones, como son los relativos a la administración, los gastos de adquisición sobre primas directas, así como

CUADRO 5. México, Primas Directas de Vida, Accidentes y Enfermedades, Daños como \% del PIB

\begin{tabular}{lllllllll}
\hline \hline & 1984 & 1985 & 1986 & 1987 & 1988 & 1989 & 1990 & 1991 \\
\hline Daños & 0,66 & 0,07 & 0,73 & 0,71 & 0,68 & 0,71 & 0,65 & 0,74 \\
Vida & 0,19 & 0,23 & 0,3 & 0,33 & 0,42 & 0,43 & 0,42 & 0,47 \\
Acc. y Enf. & 0,02 & 0,02 & 0,02 & 0,03 & 0,05 & 0,07 & 0,09 & 0,11 \\
\hline \hline
\end{tabular}


CUADRO 6. Crecimiento de la Participación por Ramo, México 1984-1993

\begin{tabular}{lrrrrrrrrrr}
\hline \hline & 1984 & 1985 & 1986 & 1987 & 1988 & 1989 & 1990 & 1991 & 1992 & 1993 \\
\hline Vida & 91,16 & 91,9 & 92,46 & 92,4 & 90,04 & 86,34 & 84,77 & 80,9 & 80,48 & 80,92 \\
Acc. y Enf. & 8,84 & 8,1 & 7,54 & 7,6 & 9,96 & 13,66 & 15,23 & 19,1 & 19,52 & 19,08 \\
\hline \hline
\end{tabular}

garantizar su solvencia (capacidad que tienen las aseguradoras para hacer frente a sus compromisos), su respaldo financiero, eficientar su capacidad de cobranza, etc., con el objeto de ser competitiva frente al mercado internacional al cual el país se abre (Cuadro 6).

Cabe resaltar que, a casi un año de la firma TLC, se anunció "la opinón favorable" para que operaran doze empresas aseguradoras extranjeras en México, a partir del primer trimestre de 1995, de las cuales seis son nortemaericanas, cinco europeas y una japonesa (La Jornada, 1994) (Es posible que esta "opinión favorable" se haya modificado a partir de la devaluación y la crisis financiera de principios de este año).

La industria nacional también se plantea como una tarea prioritaria la de promover el use del seguro a través de campañas publicitarias informativas y de sensibilización, con miras a combatir la falta de "cultura de seguro" que prevalece entre la clase media mexicana, to que sin duda será reforzado por las empresas extranjeras.

Las principales conclusiones que se pueden obtener del análisis anterior son: a) existe un crecimiento de la industria aseguradora en México, Chile, Argentina y Brasil, siendo más dinámico el de los primeros dos países; b) se registra un crecimiento sostenido de la industria aseguradora en México, en los últimos años, dentro del cuál destacase el crecimiento del ramo de accidentes y enfermedades, y; c) aunque los seguros médicos tienen una participación porcentual baja frente a los seguros de vida, al analizar su velocidad de crecimiento, observamos que es el ramo que ha crecido más rápidamente en los últimos ocho años.

\section{REFLEXIONES FINALES}

Dado que, en América Latina, los procesos de privatización de los servicios de salud se han dado en condiciones de una creciente desigualdad social y frente a una ciudadanía inmadura, dichos procesos apuntan hacia una inequidad también creciente.

Los actores sociales que han venido participando en este proceso son fundamentalmente las diferentes fracciones de la clase dominante y, hasta el levantamiento, en Chiapas, de enero de 1994, habían estado ausentes, por to menos en México, los diferentes grupos de las clases populares en este proceso. Por su parte, las organizaciones profesionales y gremiales de la salud han empezado recientemente a participar en luchas que trascienden sus intereses más inmediatos.

Asimismo, la política de ajuste se tradujo en un grave deterioro de la calidad de los servicios de las instituciones públicas, reforzando la preferencia de amplios sectores de la población por la práctica médica privada, to que constituye un importante incentivo para el incremento de los seguros médicos privados.

Dentro de este contexto, ante el escenario de conflicto político de nuestro país, la lucha colectiva por la salud ocila entre una lucha estrictamente individual de ciudadanos que han carecido históricamente de una cultura de participación política y la posibilidad de un estallamiento social de grandes magnitudes, en el que el derecho a la atención médica y a mejores condiciones de vida ocupan un lugar fundamental. En cuanto a la política gubernamental, hasta antes de la devaluación del peso mexicano, el horizonte para el presente sexenio apareció esbozado en el texto "Las políticas de Bienestar", aparecido durante la campaña de Ernesto Zedillo (1994). Llama la atención que una de las diez propuéstas consideradas como fundamentales para propiciar el crecimiento económico consiste en la necesidad de invertir en salud, educación y capacitación, 
concediendo especial importancia a la "inversión en las personas". Dentro del marco de una propuesta neoliberal sobre bienestar social, se puede entender, tal como lo interpreta Aboites (1994), que dicha propuesta entraña una concepción muy particular de bienestar social, en la cual dicho bienestar descansa en la habilidad y capacidad que cada mexicano tenga para incorporarse competitivamente en las actividades productivas para devengar el ingreso suficiente que le permita acudir al mercado y acceder a los bienes del bienestar.

En el contenido de los diez puntos planteados en el programa de salud de Ernesto Zedillo, se observa continuidad con los dos sexenios precedentes, con una profundización de los rasgos de política social que han llevado el país a una situación de aguda inestabilidad social, económica y política, lo cual se refleja en el hecho de que casi el 50\% de la población se encuentran en situación de extrema pobreza.

En la propuesta se puede apreciar un fuerte contenido privatizador, que se expresa en los siguientes aspectos: se hace especial énfasis en el fortalecimiento del mercado de servicios médicos, en el que dice a la letra: " $E s$ necesario atender los factores que inihiben la formación de un mercado más amplio de servicios médicos. El Estado deberá proponer los criterios para que esa decisión y la consolidación de este mercado estén amparados por un marco reglamentario adecuado" (Zedillo, 1994).

En el documento, también se hace referencia a la calificación de la competencia de instituciones y profesionistas, lo que en los hechos significa contar con mecanismos de evaluación que permitan flexibilizar las relaciones laborales, las condiciones de trabajo en general y, de manera muy particular, el salario de los trabajadores de la salud.

Además, se hace énfasis en la racionalización en el use de insumos y recursos y se habla de la necesidad de fomentar "la participación del sector privado en la prestación de los servicios que sean susceptibles de ser subrogados a través de licitaciones competitivas" (Zedillo, 1994).
En el futuro, este planteamiento podría implicar cambios en condiciones de abasto que ocasionen un acceso aún más diferenciado que en la actualidad a los medicamentos y, en general, a los insumos de los servicios de salud. En el desarrollo programático de este punto sería de esperarse la subrogación de servicios de laboratorio y gabinete a empresas privadas, asi como el aumento del contratismo, con la consecuente disminución de la materia de trabajo por parte de los sindicatos.

En otro apartado, se señala, como ejemplo de racionalización organizativa, la necesidad de incentivar la atención ambulatoria como alternativa a la hospitalización, así como la importancia de incentivar esquemas de atención supervisada en el hogar y la comunidad, lo que significa abaratar los costos de atención por hospitalización. Dadas las condiciones en la que viven los sectores pobres de la población, éste procedimiento frecuentemente se traduce en serios riesgos para la salud.

Retomando el análisis hecho a lo largo de este trabajo, se puede esperar una política estatal muy contradictoria, destinada, por un lado, a legitimar al gobierno, pero, por otro lado, con la necesidad impuesta por los Estados Unidos de facilitar la expansión del sector privado, a expensas fundamentalmente del crecimiento de los seguros médicos y de la privatización de los servicios de las instituciones de salud suceptibles de generar ganancia, al mismo tiempo que se da un creciente deterioro del sector público.

Este panorama se plantea dentro de una situación en la cual el principal escenario es un país en virtual estado de guerra y viviendo la crisis más profunda de su historia reciente, con una aguda reducción de los niveles de bienestar de grandes sectores de la población. Esta situación apunta a una agudización de la desigualdad social y de la inequidad, dentro de la cual la reducción del acceso a servicios de atención médica contribuirá de forma importante.

Varias preguntas podrían servir como corolario para esta artículo y tal vez para cualquier análisis que pretenda explorar algún 
aspecto relevante de la política social mexicana. ¿Qué sucederá durante este periodo de gran inestabilidad social y política, que fue denominado suntuosamente de transición democrática?; ¿hasta dónde la convulsión política que estamos viviendo cristalizará en mayor capacidad de avance de la sociedad civil en aras de disminuir la injusticia social?; ¿hasta cuándo la clase política en el poder asumirá que con el proyecto de país impulsado hace ya dos sexenios atentan contra la salud y la vida de muchos mexicanos?.

La respuesta que se de a estas preguntas, y a muchas otras que serían de fácil formulación, está por definirse, ya que se encuentran inscritas en un panorama altamente conflictivo y de redefinición de fuerzas y grupos de poder.

\section{RESUMEN}

TAMEZ, S; BODEK, C. \& EIBENSCHUTZ, C. Lo Público y Lo Privado, Las Aseguradoras y La Atención Médica en México. Cad.

Saúde Públ., Rio de Janeiro, 11 (4): 579-587, oct/dec, 1995.

Desde finales de la década de los setenta y a principios de la de los ochenta, tanto en nuestro país cuanto en la mayoría de países de América Latina, ocurrieron profundos cambios en la política sanitaria, cuya dirección apuntó fundamentalmente a un crecimiento del sector privado de la atención médica, acompañado de deterioro del sector público. Este trabajo pretende analizar, como parte del proceso privatizador de la atención médica, la evolución de los seguros médicos privados. Interesa fundamentalmente realizar un análisis de la política sectorial de salud basada en datos económicos, pero subrayando el perfil político y de modificación de las relaciones del sector con el Estado, partiendo de la hipótesis de que éste último ha asumido la función de apoyo a la expansión del mercado privado de la atención médica, enfocando el análisis a la industria de seguros médicos privados y facilitando el deterioro del sector público a través de la reducción del gasto público. Para ello se analiza el papel del Estado en las transformaciones del sector en México a partir de los principales cambios en la normatividad de la actividad aseguradora nacional. Sobre esta base se hace el análisis comparativo de la evolución de la industria aseguradora en Argentina, Brasil, Chile y México durante el período 1986-1992 profundizando el caso de México. Dentro de los principales resultados destaca que, al comparar los países mencionados, los índices Primas/PIB y Primas/ per cápita en términos generales se observa crecimiento. Este crecimiento es más ascelerado en México, lo que sin duda se relaciona con lo incipiente de su proceso de privatización. Además, en México se observó que, para el período 1984-1991, las primas directas como porcentaje del PIB pasaron de $0.86 \%$ a $1.32 \%$. En la misma línea de análisis llama la atención que el seguro de accidentes y enfermedades creció del $0.02 \%$ del PIB en 1984 a $0.11 \%$ en 1991 . En cuanto a los ramos de seguros encontramos que el rubro enfermedades y accidentes pasó de representar el 8.84\% en 1984 a 19.08\% en 1991. Esta situación expresa que el proceso de privatización de la atención médica en México tiene como uno de sus principales componentes la industria aseguradora, lo que se manifiesta a través del apoyo que el Estado ha brindado a la expansión de los grandes complejos médico industriales del país. Esto, ubicado en un contexto de continuidad del. modelo neoliberal, conducirá a una profundización de la inequidad y la desigualdad social en México.

Palabras Clave: Público; Privado; Privatización; Atención Médica; Seguros Médicos 


\section{REFERENCIAS BIBLIOGRAFICAS}

ABOITES, H., 1994. La Educación que Viene del PRI. El Financiero, Análisis-Enfoques, 10 de octubre de 1994, p. 38-A, México.

AMIS (Asociación Mexicana de Instituciones de Seguros A.C.),1991. Informe anual 1991. México. 1992. Panorama de la actividad aseguradora en México (1984-1991). № junio, México.

ASOCIACION MEXICANA DE INSTITUCIONES DE SEGUROS A.C. SECTOR SEGUROS, 1991.

Posición del sector seguros ante las negociaciones del Tratado de Libre Comercio. México: AMIS.

AVILES-TORRES, I. \& ESPINOSA-CASTELLANO, H. 1991. Organización y funcionamiento de la comisión nacional de seguros y fianzas. Actualidad en Seguros y Fianzas (México), 1: 05-17.

Diario Oficial de la Federación del 24 de febrero, 27 y 29 de marzo, 4 de abril y 4 de mayo de 1992.

EIBENSCHUTZ, C., 1991. El nuevo Estado mexicano y el sistema nacional de salud. Limitaciones para su consolidación. Saúde em Debate, 32: 33-38.

FRENK, J., 1992. Integración de la prestación de los servicios de salud. Salud Pública de Mexico, 34 (Supl.): 85-102.

GIL-ANTON, J. \& SOLIS-SOBERON, F., 1991. El Proceso de desregulación y apertura del sectorasegurador mexicano. Actualidad en Seguros y Fianzas. (México), 1: 27-36.
LAURELL, A. C., 1994. La salud: de derecho social a mercancia. In: Nuevas Tendencias y Alternativas en el Sector Salud (A. C. Laurell, org.). México: Universidad Autónoma de México.

LOVE, W. D., 1992. El Tratado del Libre Comercio y sus Repercusiones en la Industria Aseguradora. Life Insurance Market Information and Research Association. México, Mayo 13 (Mimeo.).

RUIZ-RAMIREZ, T., 1992. La Industria del Seguro ante la Integración Económica. México, 13 de mayo (Mimeo.).

SOBERON, G; DE WITT-GREENE A. \& HERNÁNDEZ ILLEZCAS, 1989. Los hospitales de segundo nivel en el modelo de atención para la salud de la población abierta. Salud Pública de Mexico, 31: 346-369.

WHO (World Health Organization), 1991. Interregional Meeting on the Public/Private Mix in National Health Systems and the Role of Ministeries of Health. Report. Hacienda Cocoyoc, State of Morelos, Mexico, 22-26 july. WHO/SHS/NHP/ 91.21 .2 (Mimeo.).

ZEDILLO, E., 1994. Las políticas del Bienestar. Documentos. México: Partido Revolucionario Institucional (Mimeo.). 